\title{
Classification Of Association Item Sets From Large Data Sets Based On User Awareness Using Hybrid
}

\author{
Srihari Varma Mantena, CVPR Prasad
}

\begin{abstract}
In business intelligence, large number of data to be generated because of increasing data in business applications. Analysis and prediction of data is very aggressive concept to evaluate the results present in data based on decision making analysis. To provide effective analysis of data traditionally some of the machine learning related methods like Clustering, Classification, Neural network based approaches and association rule based approaches were used to explore and analysis of business data. Because of increasing depth analysis of data in business intelligence related applications then above static machine learning approaches were not satisfied to form association between different attributes in real time data sets. So that in this paper, we propose Advanced \& Hybrid Machine Learning Approach (AHMLA) for effective data analysis of different associated attributes of high dimensional data. Our proposed approach increase customer service, report generations based on user awareness in business intelligence applications. An experimental result of proposed approach gives better high performance with respect to different parameters with respect to existing approaches.
\end{abstract}

Index terms: Business Intelligence, Information Retrieval, Attribute Classification, Services of customers, and Customer report generations

\section{INTRODUCTION}

In business intelligence, information retrieval is the main intellectual concept in business related applications. Data quarrying is a way of exploring records commencing different business organizations through utilization of data with different attributes present in data sources. Based on user knowledge user concealed with different association rules. Business intelligence related applications, relationship rule excavating is one of main task in user awareness to innovation \& analyzing large data sets in different business oriented applications with different data processing present in transactional data sources.

To define data implication between different data sources, association is interesting measure, association between two data sources $\mathrm{X} \& \mathrm{Y}$ i.e. $\mathrm{X} \square \mathrm{Y}$ defined by two interesting parameters like Confidence \& support, where $\mathrm{X} \& \mathrm{Y}$ are item sets \& $X \cap Y=\phi$. Apriori [1] is a to begin with calculation method to define above different association

Revised Manuscript Received on August 14, 2019.

Srihari Varma Mantena, Dept of CSE, Acharya Nagarjuna University, Guntur, AP, India(email ID: vasista4u@ gmail.com)

Dr. CVPR Prasad, Dept of CSE, Acharya Nagarjuna University, Guntur, AP, India(email ID: prasadcvpr@gmail.com) between different data items in data sources \& then some of the other calculation methods are proposed from Apriori. For efficient relation between data sources reminder rules are maintain threshold established upkeep \& confidence. Mining algorithms that can find the different association rules based on several types of attributes with respect to different types of transactions. To extract valuable information is often from unwanted association rule mining, which is suspected to presented users because of low support efficiency in transactional data sources. If we want to increase threshold value then more \& efficient algorithms $\&$ more finding rules are required and then they were helped to define association procedure and interesting to end user. Finally, it is required to maintain low support value indirection to remove useful data, values consists large volume of rules; it is not comfortable for decision making to analyze data mining result. For efficient decision making with an efficient approach to reduce association rules is an aggressive concept as a result. To solve this problem, different techniques are proposed in literature, from one perspective, diverse calculations were acquainted with diminishing the quantity of thing sets by making shut [4], leading [5] or faultless thing arrangements [6], \& a few checks to diminish a proportion of guidelines, exhausting non-excess benchmarks [7], [8], or snipping frameworks [9]. Then again, post-processing techniques can enhance the determination of found principles. Extraordinary reciprocal post-processing techniques might be utilized, such as pruning, outlining, gathering, or perception [10]. Cropping comprises happening evacuating unexciting or excess procedures. Trendy condensing, brief arrangements of standards are created. Gatherings of guidelines are created in the gathering procedure, \& the perception enhances the lucidness of an extensive number of principles by utilizing adjusted graphical representations based on different data sources.

Conventionally different methods were introduced based on random attribute analysis in database, to extract in-depth data analysis by considering user interestingness is not possible in these traditional techniques. In different types of business intelligence applications like semantic web data extraction and data analysis related applications, User knowledge is an aggressive relation in selection attributes

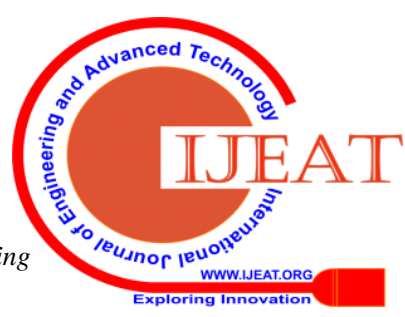




\section{CLASSIFICATION OF ASSOCIATION ITEM SETS FROM LARGE DATA SETS BASED ON USER AWARENESS USING HYBRID}

from data items. The more extract user information in an expressive \& formalize presentations based on different rules selection is efficient. In data extraction, several types' data mining applications were introduced to define user specifications. For effective data analysis in business intelligence oriented applications. In this paper, we propose Advanced \& Hybrid Machine Learning Approach (AHMLA) (which consist Association rules \& Classification rules) for effective analysis of large data sets to explore semantic $b$ data extraction \& visualization. For that, first, we propose domain User Knowledge to integrate the user knowledge in post processing representation. Secondly, we propose rule schema classification by extending user beliefs $\&$ expectations use of the data examining task.

\section{PRELIMINARIES}

For efficient data exploration, association \& classification rules expressed with different attributes. Let us consider $K=\left\{k_{1}, k_{2}, \ldots . k_{n}\right\}$ be set of items i.e. literals, \& let be group of communications on KA of $\mathrm{K}$ is thing set $\&$ it is described $X=\left\{k_{1}, k_{2}, \ldots . k_{n}\right\}$, in this item set, number of item sets are analyzed based on transactions are utilized in different item sets. Each transaction (ti) consists different items like $k_{1}, k_{2}, \ldots . k_{n}$ with n number of items for each transactions t. Relationship between data items based on classification \& association between attributes in large data sets organized with different Descriptions:

Description 1: let $X \subseteq K \& D \subseteq T$, based on these relations, define set of transactions with different item sets $\mathrm{X}$.

$$
t=K \rightarrow T, t(X)=\{t \in D \mid X \subseteq t\}
$$

Similarly, describe different item sets combined with transactions $\mathrm{T}$ by

$$
k: D \rightarrow K, i(T)=\{x \in K \mid \forall t \in T, x \in t\}
$$

Description 2: An expressive function where different attributes like where $\mathrm{X} \& \mathrm{Y}$ are two specific things, the past, $\mathrm{X}$, is known as the precursor of the idea, \& the last referenced, $\mathrm{Y}$, is known as the major. An idea $\mathrm{X}->\mathrm{Y}$ is depicted with various quantifiable information factors.

Group associated item sets, it is defined as $\sup (X \rightarrow Y)=\sup (X \cup Y)=\mid t(X \bigcup Y) \quad$ convenience ration of different attributes contains $X \cup Y$, if $\sup (X \rightarrow Y)=s$ then s\% of different attributes contain relative item sets with union relation $X \cup Y$.

Description 3: Let us consider R1, R2 two different business organizations with different data relations, we can say that $\mathrm{R} 1$ is more relative attribute $\mathrm{R} 2$, denoted by $\mathrm{R} 1 \mathrm{R} 2=\rightarrow \mathrm{R} 2$ can be explored to include different products referred with major relation $R 1$ between $R 2$, then $R j$ is the redundant, if you can relative concept $R i$ such that $R i R j$ with different relations.

Description 4: A guiding set is maximum with regard to an interestingness measurement if it is contain the entire guidelines expert from higher interestingness with other attribute relations present based on common guidelines. An ideal attribute set is the part of a non-out of work concept set.
Description 5: Totally, a Customer Information is a quintuple $\mathrm{O}=\{\mathrm{C} ; \mathrm{R} ; \mathrm{I} ; \mathrm{H} ; \mathrm{A}\}[6] . \mathrm{C}=\{\mathrm{C} 1 ; \mathrm{C} 2 ; \ldots ; \mathrm{Cn}\}$ is a lot of considerations and $\mathrm{R}=\{\mathrm{R} 1 ; \mathrm{R} 2 ; \ldots ; \mathrm{Rm}\}$ is a lot of affiliations depicted over musings. $\mathrm{I}$ is an enormous proportion of states of experiences and $\mathrm{H}$ is an Instructed Acyclic Chart (DAG) focused on the assurance respects (isa respects,) between contemplations. We express that $\mathrm{C} 2$ isa $\mathrm{C} 1, \mathrm{C} 1 \mathrm{C} 2$ if the idea $\mathrm{C} 1$ subsumes the idea C2.Ais a monstrous proportion of clichés offering extra impediments on the Customer Knowledge.

\section{REVIEW OF BACKGROUND WORK}

We analyze information available in BPI area \& discuss its co-relation with the strategy suggested in this document. In this demonstration, we analyze the related work in the following categories: work-flow record control, work-flow exploration, and work-flow managing \& marketing.

Several studies deal with the technological features considered necessary for signing the review pathway information in Zhi-Gen $\mathrm{Hu}$ et al. [1]. Huang Lei et.al [2] addressed the problem of work-flow record control in exclusive businesses in data mining. In order to provide a simpler framework as an affordable solution for businesses and discuss about health-care business intelligence by $\mathrm{V}$. Santhosh Kumar et.al [3], they recommend reducing the work-flow record by saving the chosen \& aggregated information. In order to achieve this objective, they increased the workflow specification (expressed in terms of condition and action charts) by means of orthogonal condition mapping elements \& corresponding actions for record monitoring. In this way, the process of searching the performance of a workflow can itself be seen as a workflow. Yeol Song et.al, Steven A et.al, Gan Shan et.al and Li $\mathrm{Da} \mathrm{Xu}$ et.al [4][5][6] and [7] address the business intelligence over big data in online analytical process and also discuss about different business approaches \& practical business intelligence frameworks in Mohan $S$ et.al [8] The model Mentor-lite utilizes record control as a workflow on the top of a light and portable kernel. A strategy for the monitoring record information in an allocated work-flow control system has also been provided in Tom E et.al [9]. The contributors focused on the framework and querying of the historical past in a fully allocated framework that is in conformity with Object Management Architecture (OMA) in Narasayya, $\mathrm{V}$ et.al [10] . As a part of this approach, several techniques were employed to analyze concerns against the work-flow record as shown along with their comparative execution price. Gopal A et al. [11] focused on the information warehouse design, centered on a standard work-flow meta-model that specifies the relation between work-flow specification description \& work-flow performance features.

Data exploration in work-flow records for uncovering different types of information about the workflow instances is discussed in several documents, such as [2-5] (a specific study of this research area is provided in [6]). G. Acampora [13] \& Eva Sciacca [12] \& D. P. Filev et al. [14] that also 
recommend methods for instantly deriving the official design of an activity from a log of events related to the accomplishments of a procedure that is not supported by an activity control system. However, the work is restricted only to successive procedures. Deriving from the same kind of procedure records, M. Kafai [15] , Hsinchun Chen [16] \& E.W.T. Ngai a et.al [17] obtained a work-flow design that depended on Petri nets \& integrate time details like small, maximal \& regular time placed at a certain level of the procedure. At this moment, the details get connected to places of the produced Petri net-based work-flow design. They also recommend a XML-based structure for storing \& trading work-flow records. Amir G\&omi et.al [18] \& Alam et al. [19] \& Wu et al. [20] present an inductive learning component, used to support purchase \& adaptation of successive procedure designs apart from generalization of execution records from different work-flow instances to a work-flow design while protecting all records. Our strategy differs significantly from the study projects \& expert products as presented above. We recommend an integrated strategy as well as a device package for procedure research, forecast, monitoring, management and marketing. Beyond the verification functions offered by expert products, our device supports semantic research on methods from a business perspective by changing low-level actions into company-level statistics. Another unique function is the use of details discovery methods for building forecast models. These designs are used for practical procedure management.

\section{DESCRIPTION OF PROPOSED APPROACH}

The proposed work in this paper is Advanced \& Hybrid Machine Learning Approach (AHMLA) to analyze user knowledge based on different item set formations and associations with semantic data analysis. Proposed approach (Association and Classification) consists mainly two steps appeared in figure 1 . To begin with, the learning base permits formalizing client data and destinations. Area information offers a common perspective of customer information in the database domain \& customer objectives show the first user knowledge of the found guidelines.

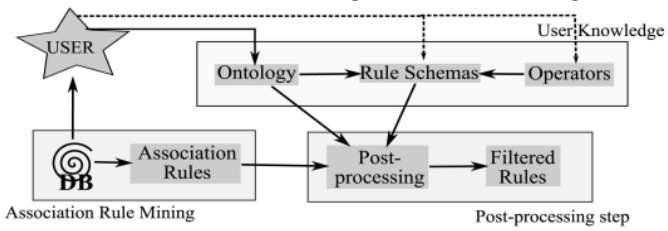

Figure 1. Hybrid approach procedure for analyzing data.

Novelty in this approach is supervising the user knowledge finding process based on conceptual structures for useful knowledge representation with several User Knowledge's, a couple of standard outlines \& summing up the impressions and dealing with an iterative representation. Procedure of the hybrid data mining for interactive process $\&$ analyzing data consists following modules.

\section{A. Post-processing Interactive Process}

Hybrid approach proposes user interactive process to discovery rules shown in figure 2 by taking user feedbacks to revise functional attributes to contribute intermediate data analyzed results.

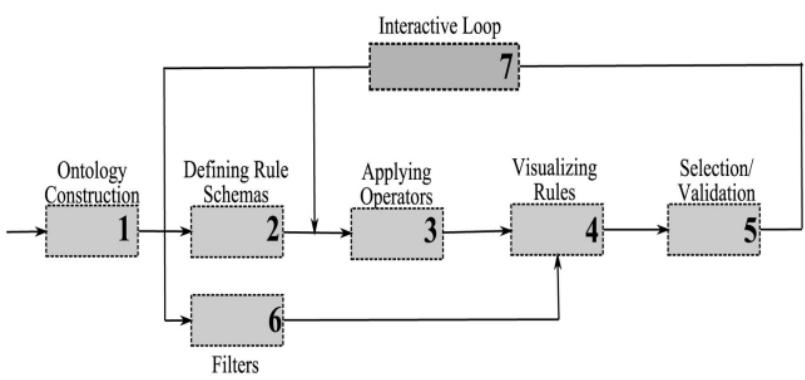

Figure 2. Interactive process description for different operators.

1. The following several steps are required to do hybrid data mining approach effectively.

2. Development of user knowledge _beginning from the database, $\&$ as time goes on, from existing ontologies, the client builds up metaphysics on database things;

3. Characterizing Rule Outlines (as GIs \& RPCs) the client communicates his/her nearby objectives \& desires regarding affiliation decides that he/she needs to discover;

4. Picking correct administrators to exist connected over the administer outlines made, $\&$ after that, applying the administrators;

5. Envisioning the outcomes_the separated affiliation rules are proposed to the client;

6. Choice/approval_beginning from these preparatory outcomes, the client can approve the outcomes or he/she can reexamine his/her data;

7. We propose to the client two channels effectively existing in the writing. These two channels can be connected over tenets at whatever point the client needs them with the fundamental objective of decreasing the quantity of standards; \&

8. The intuitive circle licenses to the client to change the data that he/she proposed. In this manner, or he/she can come back to stage 3 with a specific end goal to change the administrators.

9. In addition, in the intelligent circle, the client could choose to remove one of two _predefined channels examined in stage 6 .

\section{B. Improved Impressions based on User Knowledge}

Impressions are generally represents vague feelings of user based on precise knowledge in different data analysis with different attributes. It is very flexible, formalize \& comprehensible for every user based on different representations. General syntax for effective impressions in user view with respect to support and confidence

$$
g i\left(<S_{1}, \ldots . . S_{m}>\right) \text { [sup, conf ] }
$$

Where $\mathrm{Si}$ is a feature of products categorization or an manifestation described exhausting $*_{+}=/$? Providers \& assistance $\&$ assurance limits are optionally available. For best impressions in generally, proposed approach consists following rules.

- Accommodating standards - affiliation decides that are adjusting to the predefined convictions;

- $\quad$ Surprising forerunner rules-affiliation decides that are unforeseen in regards to the predecessor of the predefined convictions; 


\section{CLASSIFICATION OF ASSOCIATION ITEM SETS FROM LARGE DATA SETS BASED ON USER AWARENESS USING HYBRID}

- Unforeseen subsequent guidelines-affiliation decides that is surprising with respect to the subsequent of the determined convictions; \& Both side surprising standards - affiliation decides that are startling in regards to both the forerunner $\&$ the resulting in the predetermined convictions.

To enhance affiliation control determination, we propose another control sifting prototypical, entitled Rule Schemas (RS). The manage pattern depicts, in run like formalism, client desires in terms of fascinating/clear principles. Accordingly, Rule Mappings go about when in doubt gathering.

A imperative representation conveys reality that consumer believes definite components to be accompanying in the extracted suggestion guidelines. This can exist indicated as

$$
R S\left(<X_{1}, X_{2}, \ldots . . X_{n}(\rightarrow) Y_{1}, Y_{2}, \ldots . . Y_{m}>\right)
$$

Where $\quad X_{i}, Y_{j} \in C \quad$ off $\quad \% \quad O\{C, R, I, H, A\}$ repercussion "--->” is optionally available. Basically, we can remember that the proposed formalism brings together Common Opinions and Reasonably Precision Concepts (RPC).

\section{a. Description of Association Mapping}

To describe user knowledge effectively concerning in data base in our proposed approach. In this condition, it is major to interface control assessments $\mathrm{C}$ of $\mathrm{O}=(\mathrm{C} ; \mathrm{R} ; \mathrm{I} ; \mathrm{H}$; A) to the database, all of them being associated with one/a couple of things of I. To this end, we consider three sorts of assessments: leaf-evaluations, summed up bits of data from the subsumption collusion _ $(<)$ in $\mathrm{H}$ of $\mathrm{O}$, and obstruction considerations proposed just by ontologies.

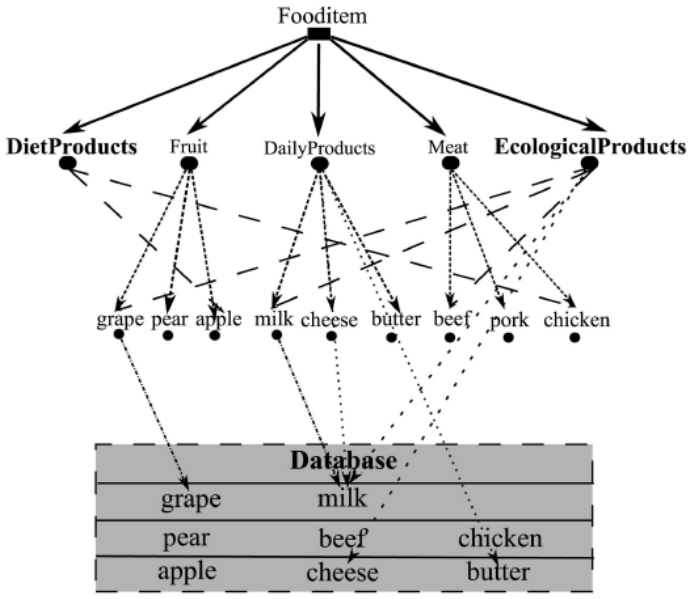

Figure 3. Description of different item sets in User Knowledge.

Protection in mind an end objective to continue with the meaning of each sort of ideas, let us help that a set to remember things in a database is characterized as $K=\left\{k_{1}, k_{1}, \ldots . . k_{n}\right\}$.

The leaf_ideas $(\mathrm{C} 0)$ are characterized as

$$
C_{0}=\left\{c_{0} \in C \mid \mathrm{h} c^{\prime} \in C, c^{\prime} \leq c_{0}\right\}
$$

They are associated at all mind boggling manner to file _each thought from $\mathrm{CO}$ is identified with one thing in the folder:

$$
f_{0}: C_{0} \rightarrow I, \forall c_{0} \in C_{0}, \exists i \in K, k=f_{0}\left(c_{0}\right)
$$

Summed up Conceptions (C1) be there depicted as the considerations that incorporate different contemplations in perspective. A summed up idea is unrelated with the folder concluded its restricted thoughts with different item sets shown in figure 3 . This implies, recursively, just the leafconcepts subsumed by the summed up idea add to its database association:

$$
\begin{aligned}
& f: C_{1} \rightarrow 2^{K} \\
& \forall c_{1} \in C_{1}, f\left(c_{1}\right)=\bigcup_{c_{0} \in C_{0}}\left\{k=f_{0}\left(c_{0}\right) \mid c_{0} \leq c_{1}\right\}
\end{aligned}
$$

Restricted Considerations are depicted utilizing reasonable verbalizations portrayed over things \& are created in the $\mathrm{C} 2$ part. In a first exertion, we compose the chart of the contemplations on constrainments over characteristics open in data bases. Thusly, the detainment figured portrayed could connect with a disjunction of things.

\section{Classify Rule Schema Operations}

The thought graph channel relies on providers utilized over thought models engaging the client to organize two or three exercises over the found recommendation. We propose two critical officials: cutting and refinement providers. The purging proprietor is made out of three unquestionable regulators: forming, astounding quality, and exemption to this thought. We offer reuse the providers proposed by Liu et al.: framing and amazing quality, and we get two new providers the post-dealing with undertaking: Trimming, Verifying and Exclusions.

\section{Pruning.}

The pruning supervisor licenses to the client to dispose of social events of considerations that he/she perspectives crippling. In information source, there exist, an imperative bit of the time, correspondence between things that we consider assertion or that we starting at now know. Everything thought of it as, isn't profitable to find these interest among the found affiliations. The pruning official related over a direct movement, $\mathrm{P}(\mathrm{RS})$, kid gets out all association standards dealing with the control applying. To confine most of the resolutions dealing with a run chart, the altering official is used.

\section{Confirming.}

The accommodating administrator connected over a manage pattern, $\mathrm{C}(\mathrm{RS})$, affirms a suggestion or finds the suggestion between a few ideas.

\section{Exceptions.}

Lastly, the exemption owner is demarcated only over implicative impression schemas (i.e., RS1) \& extracts conforming guidelines based on the following new implicative concept schema: where $\mathrm{Z}$ is the item sets.

Let us consider the example to do effective data 
presentation for implicative rule schema $X \wedge Z \rightarrow Y$ where

$$
\begin{aligned}
& f(\text { Fruits })=\{\text { grape }, \text { apple }, \text { pear }\} \& \\
& f(\text { Eco } \log \text { ical Pr oducts })=\{\text { milk }, \text { grape }\} \\
& K=\{\text { grape, apple, pear }, \text { milk, beef }\}
\end{aligned}
$$

association rules are extracted based on rule schemas are as follows:

$$
\begin{aligned}
& R_{1}: \text { grape, beef } \rightarrow \text { milk, pear } \\
& R_{2}: \text { apple } \rightarrow \text { beef } \\
& R_{3}: \text { apple, pear, milk } \rightarrow \text { grape } \\
& R_{4}: \text { grape, pear } \rightarrow \text { apple, } \\
& R_{5}: \text { beef } \rightarrow \text { grape, } \\
& R_{6}: \text { milk, beef } \rightarrow \text { grape }
\end{aligned}
$$

Thus, the actual C (RS) purification the guideline.R1 \& R3, the actual Up(RS) purification the principles R5 \& R6, \& the actual $\mathrm{Uc}(\mathrm{RS})$ purification the principles R2 \& R4. The pruning proprietor $\mathrm{P}(\mathrm{RS})$ prunes the principles chosen by the conforming proprietor $\mathrm{C}(\mathrm{RS})$.

Based on above rules with different attributes are filtered $\&$ association relation between different item sets with support \& confidence shown below.

$$
\begin{aligned}
& \text { grape, pear } \rightarrow \text { milk }(\operatorname{con} f=85 \%) \\
& \text { grape } \rightarrow \text { milk }(\operatorname{conf}=90 \%) \\
& \text { pear } \rightarrow \text { milk }(\operatorname{conf}=83 \%)
\end{aligned}
$$

In our approach, we procedure the last kind of element similarity because customers are fascinated to discover association between item sets with different features, coming from different domains.

\section{SIMULATION STUDY}

Around there, we depict the preliminary examination of our proposed crossbreed data mining approach in large volumes of data based on relational attributes. For that, we use coding language as Java \& implemented tool is Net beans for designing user interface based on our requirement. After constructing user interface like super market type business organization, using our proposed approach User Knowledge association mapping addressed between different item sets. Association mapping for different items based on their properties are discussed in section 5.1.

\section{a. Associated Database Mapping}

The client builds up a philosophy on database things. For our situation, beginning from database qualities, philosophy was made by Nantes Habitat master. Amid a few conference, we examined with the master about the database ascribes \& proposed her to arrange them. In addition, we discovered other fascinating data inquiring her to build up her desires \& learning associated with database qualities with their mapping relations are shown in figure 4 .

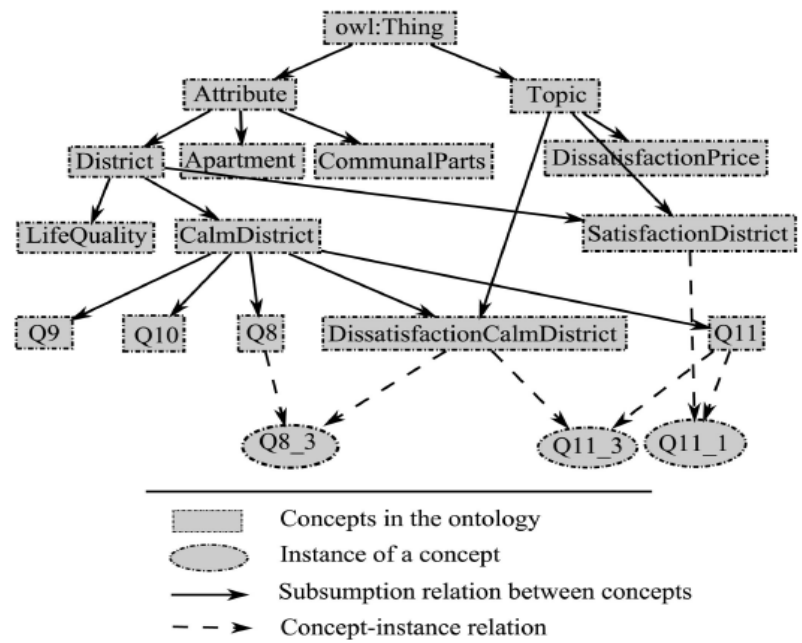

Figure 4. Association mapping relations with User Knowledge visualization.

As a piece of lead mappings, metaphysics ideas are mapped to database things. Accordingly, a few associations amongst philosophy \& database can be outlined. That is the reason coordinating a programmed metaphysics development module in our instrument is one of our important points of view.

\section{RESULTS}

This first model shows give the presentation of our new strategy concerning the loss of the wide degree of suggestions. To this end, we propose to the expert to isolate the four channels: on the either part the cutting channelsMICF, IRF, and cutting thought plans-and on nonattendance of, the choice channels-rule improvement sanitization (consequences of dense structures in work locale 1). The expert could utilize each occupy independently and in several blends to have the decision to outline the results and bolster them.

Starting now and into the foreseeable future, the ace prescribed a huge amount of cutting thought structures (Table 2) and a lot of refinement thought graphs (Table 3). Client developed these idea models during two or three social occasions of testing the new oral contraption looking outcomes.

\begin{tabular}{|l|l|}
\hline $\mathrm{Nb}$ & The id of the filter combination \\
\hline MICF & Minimum improvement constraint filter \\
\hline IRF & Item-relatedness filter \\
\hline PRS & Pruning with Rule Schemas \\
\hline Rule number & $\begin{array}{l}\text { The number of rules remaining } \\
\text { after filter application }\end{array}$ \\
\hline
\end{tabular}

Table 1. Notation meaning with descriptions 


\section{CLASSIFICATION OF ASSOCIATION ITEM SETS FROM LARGE DATA SETS BASED ON USER AWARENESS USING HYBRID}

\begin{tabular}{|l|}
\hline$R u l e$ Schema \\
\hline$R S_{5}:<$ EntryHall $\rightarrow$ CloseSurrounding $>$ \\
\hline$R S_{6}:<$ Stairwell $\rightarrow$ EntryHall $>$ \\
\hline$R S_{7}:<$ CloseSurrounding $>\rightarrow<$ EntryHall $>$ \\
\hline$R S_{8}:<$ EntryHall $\rightarrow$ Stairwell $>$ \\
\hline$R S_{9}:<$ CommonAreas $\rightarrow$ GarbageRoom $>$ \\
\hline$R S_{10}:<$ TechnicalMaintenance $\rightarrow$ TechnicalMaintenance $>$ \\
\hline
\end{tabular}

Table 2. Pruning rule schemas for different association rules.

Table 3. Filtering schemas for different relations.

\begin{tabular}{|l|c|}
\hline Rule Schema & Operator \\
\hline$R S_{1}:<$ DissatisfactionPrice $>$ & $C\left(R S_{1}\right)$ \\
\hline$R S_{2}:<$ DissatisfactionCalmDistrict $>$ & $C\left(R S_{2}\right)$ \\
\hline$R S_{3}:<$ DissasisfactionPrice & $C$ \\
DissatisfactionCommonAreas $>$ & $\left(R S_{3}\right)$ \\
\hline$R S_{4}:<$ DissasisfactionPrice $\rightarrow$ & $U_{p} / E$ \\
DissatisfactionCommonAreas $>$ & $\left(R S_{4}\right)$ \\
\hline
\end{tabular}

Based on above table's information, product information is shown in figure 5 with different item sets.

\begin{tabular}{|c|c|c|c|c|c|c|c|c|}
\hline \multicolumn{2}{|c|}{ product id product ategenty } & \multirow{2}{*}{ 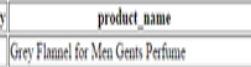 } & \multicolumn{6}{|c|}{ product_quantity unit price bullk price bullk_quantity barrode _id if id } \\
\hline ! & Coreticis & & 100 & 1.29 & 100 & 12900 & 12336 & 12356 \\
\hline 2 & Cosmetics & ARAMSS 9ON EDF' IIOML FOR MEN & 100 & 3449 & 173000 & 100 & 12338 & 12338 \\
\hline 3 & Cosetics & HugpoukBlue For Nea & 100 & 1730 & 100 & 175000 & 13358 & 12388 \\
\hline t & Cowmetis & ROYAL VIIRAGE BROWN PERELME FOR MEN & $\sqrt{100}$ & 394 & 3940 & 100 & 11339 & 12359 \\
\hline 5 & Groteis & Malk-Gold & 1000 & 20 & 1000 & 20000 & 2365 & 2366 \\
\hline 6 & Groveries & Milk Blote & 1000 & 17 & 1000 & 17000 & 23366 & 23366 \\
\hline 7 & Groteis & Mallorage & 1000 & 15 & 1000 & 15000 & 2363 & 2363 \\
\hline 8 & Grocties & Milik & 1000 & 17 & 17000 & 1000 & 22369 & 22360 \\
\hline 9 & Groteits & Bread & 1000 & 20 & 1000 & 20000 & 27360 & 27350 \\
\hline 10 & Groteris & Salt & 1000 & 10 & 1000 & 10000 & 27361 & 27360 \\
\hline III & Groeies & Oil & 1000 & $(60)$ & 1000 & 00000 & 27362 & 27360. \\
\hline 12 & Groteris & Chips & 1000 & 13 & 1000 & 15000 & 221363 & 27360 \\
\hline$\sqrt{13}$ & Groteis & Sits & 1000 & 100 & 1000 & 100000 & 27364 & 27364 \\
\hline 14 & Vegetables & Ohions & 1000 & 30 & 1000 & 30000 & 177136 & 177736 \\
\hline 15 & Vegetables & Carrots & 1000 & 25 & 1000 & 5000 & $1233 \%$ & 1333 \\
\hline 16 & Neptatiles & Bettioot & 1000 & 40 & 1000 & 40000 & 1232 & 1232 \\
\hline
\end{tabular}

Figure 5. Different product information based on items presented in business intelligence.

Using this information to construct data relations on supermarket business shown in figure 6 with suitable support \& confidence in different sequences.

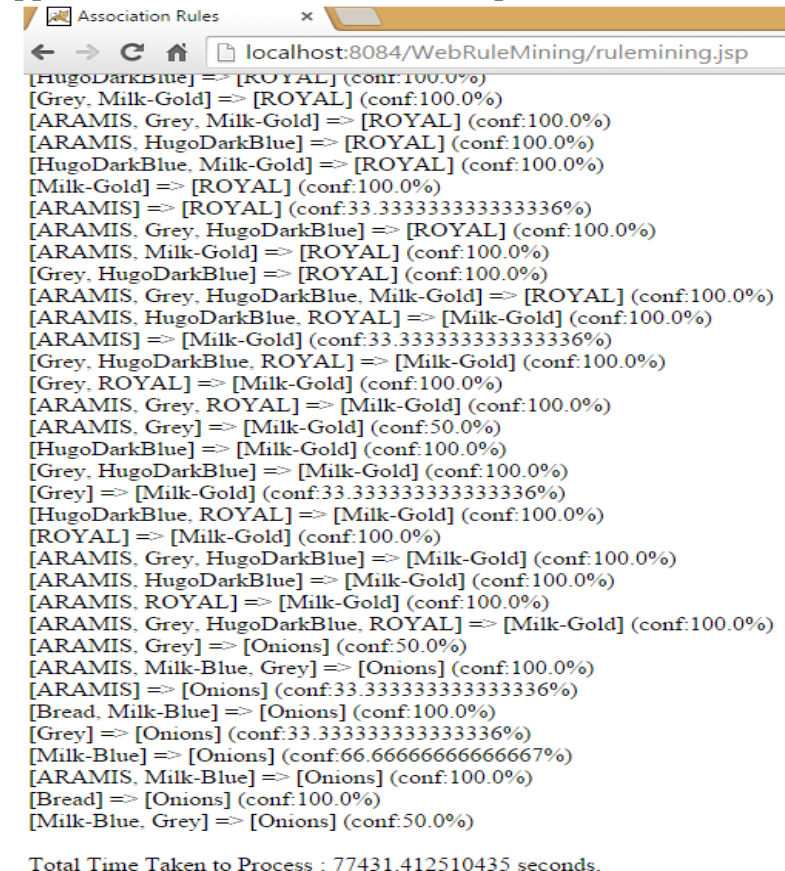

Figure 6. Association rules based on item set description with their values.
Figure 6 shows the hybrid data mining approach results, after applying classification on each data item associated rule mining sequences shown in table 2,3. Time efficiency to classify different items with relationships performance results are shown in figure 7.

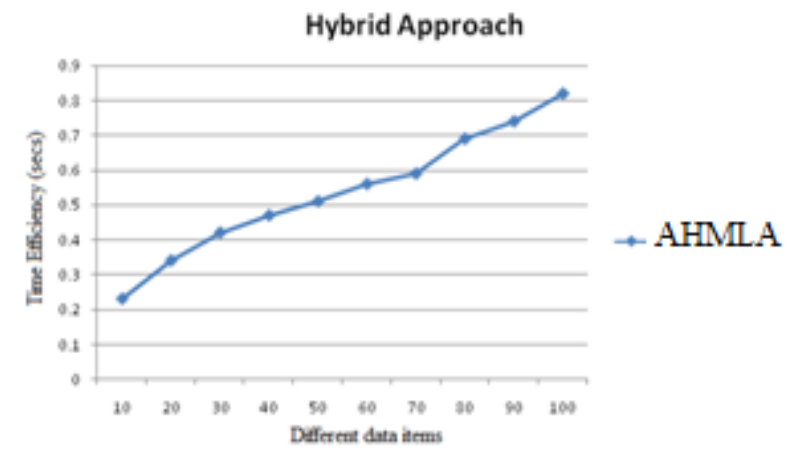

Figure 7. Time efficiency results of hybrid approach with different attributes

As shown in above figure \& tables, the proposed approach gives better sequential results for different attributes. Finally, these results give best \& effective associated attributes from different business oriented applications effectively.

\section{CONCLUSION}

In this paper, we propose \& implement Advanced \& Hybrid Machine Learning Approach (AHMLA) for an interesting relation between different item sets from large amounts of data. We analyze different relations from different attributes based on knowledge of user throughout large amounts of data evaluation. In this approach, we propose different rule schemas based on ontology description of user knowledge applicable operate user interestingness with usage of user processing. First, we retrieve user awareness with respect to association between attributes. Secondly, set of attribute relations described usage of several operations like data evaluation exceptions $\&$ other parameter processing. We also integrate in depth analysis association different attributes with support \& confidence results. Experimental results show effective formation of data with different attribute relations. Further improvement of proposed approach is to support optimize attribute relations between different item sets from large high dimensional datasets

\section{REFERENCES}

1. R. Agrawal, T. Imielinski, and A. Swami, "Mining Association Rules between Sets of Items in Large Databases," Proc. ACM SIGMOD, pp. 207-216, 1993

2. M.L. Antonie, O.R. Zaiane, and A. Coman, "Use of Data Mining Techniques for Medical Image Classification," Proc. Second Intl. Workshop Multimedia Data Mining in Conjunction with seventh ACM SIGKDD (MDM/KDD '01), 2001.

3. G. Cong, A.K.H. Tung, X. Xu, F. Compartment, and J. Yang, "Farmer: Finding Interesting Rule Groups in Microarray Datasets," Proc. ACM SIGMOD Int'l Conf. The leading group of Data (SIGMOD '04), 2004.

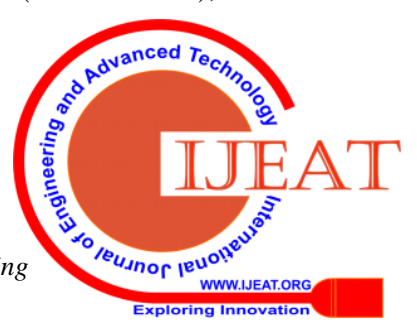


4. W. Wang, J. Yang, and P.S. Yu, "Beneficial Mining of Weighted Association Rules (WAR)," Proc. sixth ACM SIGKDD Int'l Conf. Data Discovery and data Mining (KDD '00), pp. 270-274, 2000.

5. F. Tao, F. Murtagh, and M. Farid, "Weighted Association Rule Mining Using Weighted Support and Significance Framework," Proc. nineth ACM SIGKDD Int'l Conf. Learning Discovery and Data Mining (KDD '03), pp. 661-666, 2003

6. Gan Shan, Du Haoquan," Research on Business Intelligence Application in Electric Grid Industry Based on Data Mining", appropriated in Intelligent Computation Technology and Automation (ICICTA), 2014 seventh International Conference on ieee Xplore 08 January 2015.

7. Lian Duan, $\mathrm{Li} \mathrm{Da} \mathrm{Xu}$, "Business Intelligence for Enterprise Systems: A Survey " appropriated in IEEE Transactions on Industrial Informatics ( Volume: 8, Issue: 3, Aug. 2012 ).

8. A.M. Watching out for, D.J. Haglin, and J.A. Keane, "A Recursive Search Algorithm for Statistical Disclosure Assessment," Data Mining and Knowledge Discovery, vol. 16, no. 2, pp. 165-196, http://mavdisk.mnsu.edu/haglin, 2008.

9. D.J. Haglin and A.M. Watching out for, "On Minimal Infrequent Itemset Mining," Proc. Int'l Conf. Data Mining (DMIN '07), pp. 141-147, 2007.

10. J. Han, J. Pei, and Y. Yin, "Mining Frequent Patterns without C\&idate Generation," Proc. ACM SIGMOD Int'] Conf. The officials of Data, pp. 1-12, 2000.

11. J.M. Kleinberg, "Genuine Sources in a Hyperlinked Environment," J. ACM, vol. 46, no. 5, pp. 604-632, 1999.

12. C.- K. Chui, B. Kao, and E. Hung, "Mining Frequent Itemsets from Uncertain Data," Proc. eleventh PacificAsia Conf. Advances in Knowledge Discovery and Data Mining (PAKDD '07), pp. 47-58, 2007.

13. T. Bernecker, H.- P. Kriegel, M. Renz, F. Verhein, and A. Zuefle, "Probabilistic Frequent Itemset Mining in Uncertain Databases," Proc. fifteenth ACM SIGKDD Int'l Conf. Learning Discovery and Data Mining (KDD '09), pp. 119-128, 2009..

14. D. P. Filev, R. B. Chinnam, F. Tseng, P. Baruah, "A cutting edge quality peculiarity recognizable proof framework for free rigging watching and diagnostics", IEEE Trans. Ind. Informat., vol. 6, no. 4, pp. 767-779, Nov. 2010.

15. M. Kafai, B. Bhanu, "Dynamic Bayesian frameworks for vehicle request in video", IEEE Trans. Ind. Informat., vol. 8, no. 1, pp. 100-109, Feb. 2012.

16. Hsinchun Chen, Roger H. L. Chiang, Veda C. Story, "Business Intelligence and Analytics: From Big Data To Big Impact", MIS Quarterly Vol. 36 No. 4, pp. 11651188/December 2012.

17. M. Zaki, "Mining Non-Redundant Association Rules," Data Mining and Knowledge Discovery, vol. 9, pp. 223 248, 2004.

18. A. Maedche and S. Staab, "Customer Knowledge Learning for the Semantic Web," IEEE Intelligent Systems, vol. 16, no. 2, pp. 72-79, Mar. 2000.

19. B. Liu, W. Hsu, L.- F. Mun, and H.- Y. Lee, "Finding Interesting Patterns Using User Expectations," IEEE Trans. Data and Data Eng., vol. 11, no. 6, pp. 817-832, Nov. 1999.

20. M.J. Zaki and C.J. Hsiao, "Bid: An Efficient Algorithm for Closed Itemset Mining," Proc. Second SIAM Int'1 Conf. Data Mining, pp. 34-43, 2002.

21. M.Z. Ashrafi, D. Taniar, and K. Smith, "Tedious Association Rules Reduction Techniques," AI 2005: Advances in Artificial Intelligence - Proc eighteenth
Australian Joint Conf. Mechanized thinking pp. 254-263, 2005.

22. M. Hahsler, C. Buchta, and K. Hornik, "Specific Association Rule Generation," Computational Statistic, vol. 23, no. 2, pp. 303-315, Kluwer Academic Publishers, 2008

23. J. Bayardo, J. Roberto, and R. Agrawal, "Mining the Most Interesting Rules," Proc. ACM SIGKDD, pp. 145154, 1999.

24. R.J. Bayardo, Jr., R. Agrawal, and D. Gunopulos, "Basic Based Rule Mining in Large, Dense Databases," Proc. fifteenth Int'l Conf. Data Eng. (ICDE '99), pp. 188-197, 1999.

\section{AUTHORS PROFILE}

Srihari Varma Mantena, Research Scholor, Dept of CSE, Acharya Nagarjuna University, Guntur-522510, AP, INDIA

Email ID: vasista4u@gmail.com

Dr CVPR Prasad, Research Supervisor, Dept of CSE, Acharya Nagarjuna University, Guntur-522510, AP, INDIA

Email ID: prasadcvpr@gmail.com 